\title{
Cloud Computing : Goals, Issues, SOA, Integrated Technologies and Future-scope
}

\section{Madhu Viswanatham \\ School of Computing Science and Engineering, VIT University, Vellore, India 632014}

Key words: Cloud Architecture, Business Intelligence, Mobile cloud, Cloud Storage, Services

\begin{abstract}
The expansion of networking infrastructure has provided a novel way to store and access resources in a reliable, convenient and affordable means of technology called the Cloud. The cloud has become so popular and established its dominance in many recent world innovations and has highly influenced the trend of the Business process Management with the advantage of shared resources. The ability to remain disaster tolerant, on-demand scalability, flexible deployment and cost effectiveness has made the future world technologies like Internet of Things, to determine the cloud as their data and processing center. However, along with the implementation of cloud based technologies, we must also address the issues involved in its realization. This paper is a review on the advancements, scopes and issues involved in realizing a secured cloud powered environments.
\end{abstract}

method of delivering technology. This shows it is not a recently emerged technology. The cloud computing finds it roots in early 1950's. The cloud architecture should possess the ability to be scalable, flexible, highly abstract, reliable, on-demand, remote handling, and metered usage and secure (Marston et al., 2011; Whaiduzzaman et al., 2014). The cloud is a service that has three branches popularly called IaaS model, PaaS model and SaaS model (Buyya et al., 2008;2009; Oliveira et al., 2014; Jula et al., 2014).

IaaS: The Infrastructure as a service includes Virtual Machines to access the remote hardware or to access the operating system services hosted in the cloud environment. This is facilitated by the means of an Application Programming Interface. The Infrastructure as a service includes Database as a service, Data as a service, Privacy as a service, Information as a service and Firmware as a service (Marston et al., 2011; Bruneo, 2014).

PaaS: Platform is a medium that provides facilities to the client to make eff icient use of the cloud technologies. The Client could develop his own applications and deploy in the cloud using the Cloud platform. The PaaS provides Simulation and testing of the user programs as a service.

SaaS: The Software is the integrated program that is meant to provide service and interface between the user access. The ENISA describes cloud computing as a novel

*Corresponding Author: vmadhuviswanatham@vit.ac.in 
end and the service provider end. These are developed and hosted by third parties, which can be accessed by the means of billed usage or for free. Cloud business insight was basically the area of startup merchants offering Software as a Service (SaaS) Cloud BI's potential advantages are like those offered by distributed computing innovation when all is said in done.decreased server farm and IT administration costs, quicker arrangement times, expanded adaptability as business needs change. By and large, however, cloud BI organizations are still moderately low-end in nature

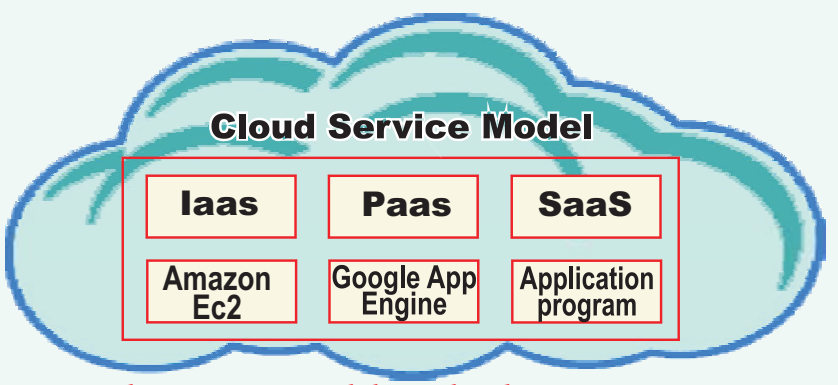

Figure-1: threeService models in Cloud Computing. Source: Branch etal., 2014

All these service models are developed to stand as an interface between the Service provider and client end. The high speed reliable Internet infrastructure counts to its performance. The Four Deployment models are

1. Public Cloud with open access to all

2. Private Cloud with restricted access to an authorized individual organization/person

3. Hybrid Cloud with the combination of distinct clouds facilitates the load balancing mechanism.

4. Community Cloud with restricted use among a group of people who belong to an authorized community.

With all these features the cloud computing establishes a new paradigm in the era of digitalization. There is no doubt that the future smart cities and smart innovations would have the contribution of cloud computing, to find stability (Mell \& Grance, 2011).

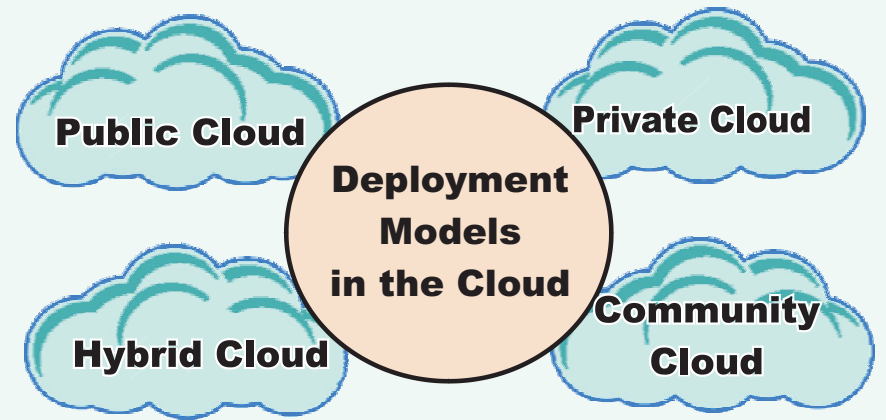

Figure-2: four Deployment models in the cloud computing

\section{Cloud Goals and Characteristics:}

The Cloud computing shows a fast growth in the world of technology. The major goals of this well flourished technology lies in the Service oriented Architecture, by the combination of the utility computing, grid computing and distributed computing, to produced increased levels of flexibility and convenience at reduced ownership cost.

The Characteristics of the cloud
1. On-demand Serviceability
2. Broad Network Accessibility
3. Shared Resource Pooling
4. Elasticity
5. Metered Service

On-Demand Serviceability: The cloud has the ability of self-service to the user end with the automation of provisioning resources on demand.

Broad Network Accessibility: Cloud computing is totally based on the Internet Infrastructure. The Internet is the gateway to exploring the world of cloud. A seamless, high-speed and reliable internetworking infrastructure is an essential characteristic to realize a reliable cloud technology. It enables the interoperability among the heterogeneous devices involved in the cloud. The client platform is characterized into two based on the workload as a thin client, which takes a little load and dumps another process to the server and the fat client, which loads the maximum task in itself and approaches the server on requirement with less workload. There are various standard mechanisms introduces to pull almost all devices into the web-based computing. There are mobile phones, tablets, PCs, wearable devices etc., enabled by the Internet Infrastructure. Thus the cloud computing is formed with these devices as the medium of access.

Shared Resource Pooling: The concept of Multi-tenancy is possible due to this characteristic of the cloud technology. A resource at the other end can be accessed from anywhere and anytime with high reliability by multiple users on the shared basis, using the Virtual Machines. The resource could be a data, software or hardware service available at a remote end, accessed on authorization and authentication.

Elasticity: The ability to expand the services and capabilities rapidly, on requirement is termed as Elasticity.

Metered Service: The services offered by the cloud could be metered based on the parameters like Bandwidth, Data Storage, Processing etc. This is a measure to establish transparency between the client and the server on resource consumption. The services offered are in free to use or pay foruse.

\section{Challenges in Cloud Computing:}

The Workflow Scheduling challenge is characterized as the most critical challenge due to its impact on the System Architecture, Quality of Service and the functionality of the Cloud system. The Cloud based WFS is based on the system model and the service driven model. 
Table-1: Various WFS algorithms and their features

\begin{tabular}{ll}
\hline No WFS method & Functionality
\end{tabular} $\begin{aligned} & \text { Type of Environment Features } \\
& \text { Approach }\end{aligned}$

$1 \quad$ Market Oriented Assurance of Suitable workflow QoS for Hierarchical Scheduling user-presented WFS functions. Robust Strategy MOHSS optimization procedures to reduce the cost of the systems running cloud work -flow methods.

2 Multiple QoS of MultiWorkflows (MQMW)

3 Business Grid Quality of Service (BGQS)

4 Cost optimization and time optimization scheduling policies (COTOSP)

5 DAG-LOSS \& DAG-GAIN

6 Multiple QoS based Resource Scheduling Algorithm (MQRS)

7 Mixed-Integer NonLinear Programming (MINLP)

8 Optimized Resource Scheduling Algorithm (ORSA)

9 Innovative transaction intensive costconstraint scheduling algorithm (ITICCSA)

10 Particle Swarm Optimization-based Heuristic for Scheduling (PSBHS)

11 Time and costconstrained scheduling strategy (TCCSS)
Multiple workflows are scheduled

which are initiated at any time by considering the QoS requirements.

Matching QoS of business applications with characteristics of heterogeneous grid resources and adjusting if necessary during execution to maintain agreed service levels acts as a broker at the user-level, hires

A fundamental framework is used for workflow applications modeled like DAGs, and heuristics that enable scheduling of DAG nodes or workflow tasks onto the resources are examined. by breaking down the cost of the task into two parts: (i) computation resource cost; and (ii) bandwidth resource cost.

Individual application details are abstracted but the global cost optimization problem and scheduling of the entire grid workload are focused on.

Execution cost and time Workflow with

Resource utilization, time Group of tasks

Dynamic, shared and autonomous resources are adopted in grids.

$\begin{array}{lll}\text { Cloud } & \text { Meta- } & \text { QoS requirement is satisf ied } \\ & \text { Heuristic } & \text { at low cost of WFS }\end{array}$

Cloud, Heuristic

Scheduling success rates are prominently enhanced. It is designed for several workflows with various QoS requirements

Grid Business Oriented

Directed to mainstream businessoriented applications, key features include: the facility toallow the Grid Resource Consumer (GRC) to request $\mathrm{QoS}$ at varying levels, e.g. time, cost, CPU or RAM; dynamically calculated metrics for measuring QoS such as reliability; matchmaking and monitoring to establish whether Grid Resource Providers (GRP) can deliver a suitable level of QoS to the GRC; and reallocation of resource during execution

The Gridbus broker is employed, which Cloud Market resources from Amazon Elastic Compute Oriented The computational capability of local resources is enhanced by obtaining resources from IaaS providers to attain the deadline within the budgetary constraints.

Grid Heuristic

Budget constraints are satisfied while time in general is optimized.

Cost is incurred when a task is executed Grid

Scheduling Parameters Speed,Resource Cloud Multiple Utilization \& Request allocation problem instances large number of instances

Cloud Batch

Mode

Cloud Dependency mode

Computational Acceptable scheduling is provided economybased framework for real-world workflows in three dimensions: (i) cost; (ii) deadlines; and (iii) reliability.

Grid HoltWinters Workflow deadlines are satisf ied at method lower cost and higher utilization of underlying grid resources to improve failure rates and turnaround times.

Speed of the IGA is almost twice the traditional GA 2.The utilization rate of resources is high.

1.To minimize the cost under certain user-designated Deadlines. 2.

Enables the compromises of execution cost and time.

1. it is used for three times cost savings as compared to BRS 2.It is used for good distribution.

Grid Extended critical activity
Less completion time and lower cost meet requirements in practical applications, which can effectively meet users' needs. 
No WFS method

Functionality

Individual application details are abstracted but the global cost optimization problem and scheduling of the entire grid workload are focused on.

8 Optimized Resource Scheduling Algorithm (ORSA)

Scheduling Parameters Speed, Resource Utilization and Request allocation problem

9 Innovative transaction Execution cost and time Workflow with intensive cost-constraint large number of instances scheduling algorithm (ITICCSA)

10 Particle Swarm Optimization-based Heuristic for Scheduling (PSBHS)

11 Time and costconstrained scheduling strategy (TCCSS)
Resource utilization, time Group of tasks

Dynamic, shared and autonomous resources are adopted in grids.
Type of Environment Features

Approach

$\begin{array}{lll}\text { Grid } & \begin{array}{l}\text { HoltWinters } \\ \text { method }\end{array} & \begin{array}{l}\text { Workflow deadlines are satisfied at } \\ \text { lower cost and higher utilization of } \\ \text { underlying grid resources to } \\ \text { improve failure rates and } \\ \end{array} \\ & \text { turnaround times. }\end{array}$

Cloud Multiple instances

Speed of the IGA is almost twice the traditional GA 2.The utilization rate of resources is high.

Cloud Batch Mode

1.To minimize the cost under certain user-designated Deadlines.

2. Enables the compromises of execution cost and time.

Cloud Dependency mode

1. it is used for three times cost savings as compared to BRS 2.It is used for good distribution.

$\begin{array}{ll}\text { Grid } & \begin{array}{l}\text { Extended } \\ \text { grid }\end{array} \\ & \text { critical } \\ \text { activity }\end{array}$

Less completion time and lower cost meet requirements in practical applications, which can effectively meet users' needs.

\section{WFS Metrics:}

The Quality of Service of the various algorithms is studied based on the three categories system functionalities and the System architecture. The scheduling of the Cloud workflow involves the cost aware approach that considers the profitability level of the consumers and the service providers. The QoS deals with the budget, Deadline, Reliability, Availability, makespan, SLA, and Security. There will be interaction among the various QoS factors to decide the cost of the cloud-based systems.

Budget: It is the threshold of the amount spent on a system.

Deadline: It is the threshold of the time for Work Flow completion.

Reliability: The ability of the system to be readily available on demand for independent allocation of the cloud resources.

Availability: The Availability improves the maintainability of the resources. This has an impact on the ability to test the resources in a workflow scheduling environment.

Makespan: It defines the overall time taken for the completion of a workflow Schedule in the Cloud Environment.

SLA: It is a legal agreement, mutually accepted by the client and the service providers that def ine the parameters of Quality, Payment, and limitations. Security: It deals with the level of trustworthiness of a cloud-based service. The confidentially is also a part of security which should ensure highly conf idential execution of the workflow.

These factors of the QoS have a direct impact on the three service models of the cloud and the servicedriven architecture. The service-driven architecture includes the Client and utility provider. The System architecture of a WFS includes the hardware and the intensiveness of the system. The Hardware-aware WFS deals with the multiple core awareness models of WFS whereas the System intensive models concentrate on the object, data, communication and the ability for multiple workflows. The System functionality includes the time complexity, rescheduling, resource utilization, resource allocation, load balancing and task estimation challenges.

\section{SOA in Cloud Computing:}

Cloud Computing comprises of a Service-oriented Architecture than an application oriented Architecture. The distributed Computing has laid the foundation for this advancement in the cloud [11]. The RPC is the first SoA in the network based systems. The key concept of Serviceoriented architecture is a component based approach. In service oriented Architecture, the client requests and the server offer the service on demand. The SoA determines the workflows. The service is provided as soon as the request is received or in some cases, it could be processed in allotted time. The fault tolerance, audit, data aware and process aware service delivery are the characteristics of a SoA architecture. Characterizes the Component-based approach by this given factors- i)Ability to reuse, ii) Ability to replace or substitute, iii) Ability to extend, iv) Ability to scale, v) Ability to customize features, vi) Ability to Compose functional solutions, vii) Ability to be reliable and available, viii) Ability to provide security, ix) Cost of services. 
Table- 2: Analysis of QoS challenges in WFS on various algorithms

\begin{tabular}{|lllllllll|}
\hline & & $\mathrm{I}$ & $\mathrm{II}$ & $\mathrm{III}$ & $\mathrm{IV}$ & $\mathrm{V}$ & $\mathrm{VI}$ & $\mathrm{VII}$ \\
\hline 1 & MOHSS & $\mathrm{X}$ & $\mathrm{X}$ & $\mathrm{X}$ & $\mathrm{X}$ & - & $\mathrm{X}$ & $\mathrm{X}$ \\
2 & MQMW & $\mathrm{X}$ & $\mathrm{X}$ & $\mathrm{X}$ & $\mathrm{X}$ & - & $\mathrm{X}$ & $\mathrm{X}$ \\
3 & BGQS & - & - & - & - & & - & $\mathrm{X}$ \\
4 & COTOSP & - & - & $\mathrm{X}$ & $\mathrm{X}$ & - & $\mathrm{X}$ & $\mathrm{X}$ \\
5 & DAG-LOSS & - & $\mathrm{X}$ & $\mathrm{X}$ & $\mathrm{X}$ & - & $\mathrm{X}$ & $\mathrm{X}$ \\
& \&DAG-GAIN & & & & & & & \\
6 & MQRS & - & - & - & $\mathrm{X}$ & - & $\mathrm{X}$ & $\mathrm{X}$ \\
7 & MINLP & $\mathrm{X}$ & - & $\mathrm{X}$ & $\mathrm{X}$ & $\mathrm{X}$ & $\mathrm{X}$ & $\mathrm{X}$ \\
8 & ORSA & $\mathrm{X}$ & $\mathrm{X}$ & - & $\mathrm{X}$ & $\mathrm{X}$ & $\mathrm{X}$ & $\mathrm{X}$ \\
9 & ITICCSA & - & - & $\mathrm{X}$ & $\mathrm{X}$ & $\mathrm{X}$ & $\mathrm{X}$ & $\mathrm{X}$ \\
10 & PSBHS & - & - & $\mathrm{X}$ & $\mathrm{X}$ & $\mathrm{X}$ & $\mathrm{X}$ & $\mathrm{X}$ \\
11 & TCCSS & - & - & $\mathrm{X}$ & $\mathrm{X}$ & $\mathrm{X}$ & $\mathrm{X}$ & - \\
\hline
\end{tabular}

Abbreviations: I-Budget; II-Deadline, III-Reliability, IVAvailability, V-Makespan, VI-SLA VII-Security

Table-3: Qos based on System functionality of various algorithms

\begin{tabular}{|c|c|c|c|c|c|c|}
\hline & I & II & III & IV & $\bar{V}$ & VI \\
\hline 1 MOHSS & - & $\mathrm{X}$ & $\mathrm{X}$ & - & $\mathrm{X}$ & Client \\
\hline 2 MQMW Provider & $\mathrm{X}$ & $\mathrm{X}$ & $\mathrm{X}$ & $\mathrm{X}$ & - & Service \\
\hline 3 BGQS & $\mathrm{X}$ & $\mathrm{X}$ & $\mathrm{X}$ & $\mathrm{X}$ & - & Client \\
\hline 4 COTOSP & $\mathrm{X}$ & $\mathrm{X}$ & - & $\mathrm{X}$ & $\mathrm{X}$ & Client \\
\hline \multirow{2}{*}{$\begin{array}{ll}5 & \text { DAG-LOSS } \\
& \& \text { DAG-GAIN }\end{array}$} & - & $\mathrm{X}$ & - & $\mathrm{X}$ & $\mathrm{X}$ & Client \\
\hline & & & & & & \\
\hline 6 MQRS provider & $\mathrm{X}$ & $\mathrm{X}$ & $\mathrm{X}$ & $\mathrm{X}$ & - & Service \\
\hline 7 MINLP provider & - & $\mathrm{X}$ & - & - & $\mathrm{X}$ & Service \\
\hline 8 ORSA & - & - & $\mathrm{X}$ & $\mathrm{X}$ & $\mathrm{X}$ & Client \\
\hline 9 ITICCSA & - & $\mathrm{X}$ & $\mathrm{X}$ & $\mathrm{X}$ & $\mathrm{X}$ & $\mathrm{X}$ \\
\hline 10 PSBHS & - & - & $\mathrm{X}$ & $\mathrm{X}$ & $\mathrm{X}$ & Client \\
\hline 11 TCCSS provider & $\mathrm{X}$ & - & - & $\mathrm{X}$ & $\mathrm{X}$ & Service \\
\hline \multicolumn{7}{|c|}{$\begin{array}{l}\text { Abbreviations: I-Time Complexity, II Resource utilization, } \\
\text { III-Resource allocation, IV-Load balancing, V-Task estimation, } \\
\text { VI-Profitability }\end{array}$} \\
\hline
\end{tabular}

Table-4: the Frequencies of the Qos Challenges are calculated and they are tabulated as follows

\begin{tabular}{|lll|}
\hline & Frequency(in \%) & Challenge \\
\hline Budget & 15 & QoS \\
Deadline & 27 & QoS \\
Reliability & 7 & QoS \\
Availability & 5 & QoS \\
makespan & 31 & QoS \\
SLA & 6 & QoS \\
Security & 9 & QoS \\
Object intensive & 16 & System Functionality \\
Data intensive & 37 & System Functionality \\
Communication & 16 & System Functionality \\
intensive & & \\
Multicore aware & 21 & System Functionality \\
Multiple workflow & 10 & System Functionality \\
Time complexity & 28 & System Architecture \\
Rescheduling & 18 & System Architecture \\
Resource utilization & 16 & System Architecture \\
Resource Allocation & 16 & System Architecture \\
Load Balancing & 10 & System Architecture \\
Task estimation & 12 & System Architecture \\
\hline \hline
\end{tabular}

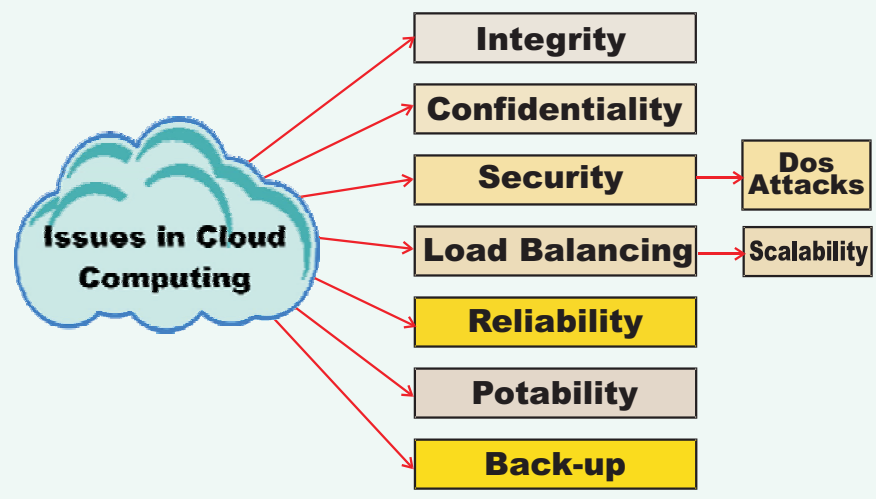

Figure- 3: the main issues in Cloud computing

The workflow scheduling is always represented using a DAG (Directed Acyclic Graph) that connects the components that are loosely coupled with the components that are tightly coupled (Abrishami et al., 2013; Jain et al., 2014). Virtualization is also a part of the SoA that provides the portability between the higher and lower ends of computing. IBM mainframe stands as the pioneer for the concept of virtualization that has been widely deployed in all forms of the computing (Buyya et al., 2008; Zhan et al., 2015).

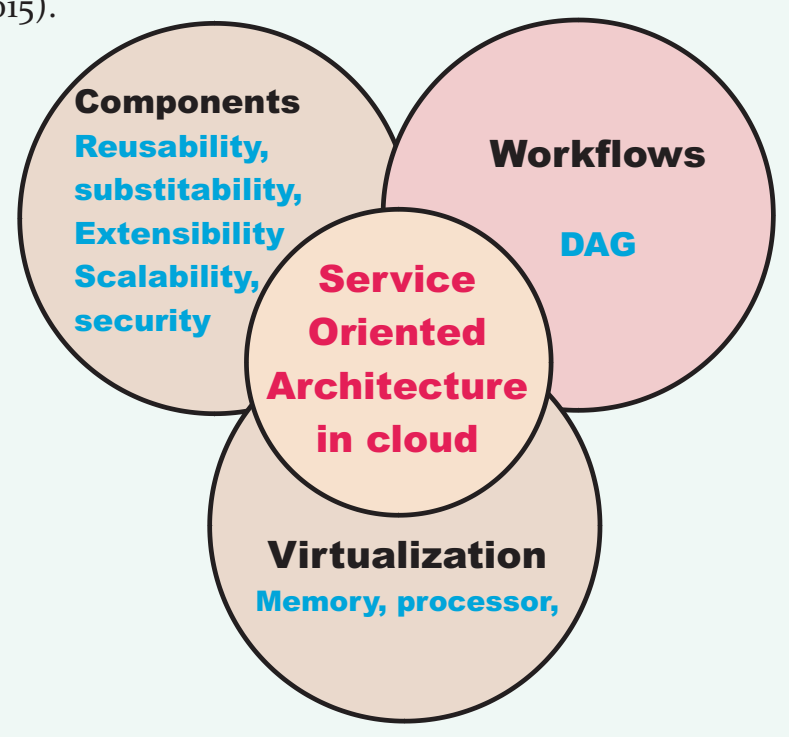

Figure-4: Service oriented Architecture in cloud computing.

\section{Adoption of Cloud Computing in Emerging Technologies:}

'Push all in Cloud and Access with Internet' is the trend set by the cloud computing Technology. The cloud computing is described as the realization of the cyberinfrastructure along with Virtualization, Grid Computing, Distributed Computing and Utility computing. Data is the basic element in the world of Internetworking (Abolfazli et al., 2015). The Data protection, processing, and storage are the most concentrated areas of research in today's world. The Data storage devices face several drawbacks (Bruneo, 2014; Dong et al., 2014). The processing of large amounts of data 
accounting to petabytes could not be solved by an ordinary physical machine. Hence, there is born an idea to migrate these important tasks to the cloud. The term big data was introduced, and the three important milestones namely Protection, Processing and Protection are achieved. The advancement in Digital Electronics has also laid a path to the technology, which is estimated as the Technology of future with its enormous growth and extensive application. The Internet of Things is a combination of the embedded systems, integrated networking technologies, mobile computing, ubiquitous computing, data Processing and storage (Wang et al., 2014; Aazam et al., 2014). The mobile computing technology has also integrated with cloud and it gave birth to the technology called MCC (Mobile Cloud Computing) with offloading facilities (Ahmed et al., 2015). Let us discuss a few emerging technologies and how they are integrated with the cloud to offer a wide range of services.

The technologies will integrate and diffuse at a stage to form a powerful integrated Technology. Internet of Things is expected to become the super powerful technology of the world in forthcoming decades. This ability is possible only because of the cloud computing. The Table (1) clearly shows the dominance of the cloud computing in the emerging technologies. Many of these technologies still remain in their infancy due to the security, load balancing, cost of computing, fault tolerance and reliability issues (Wei et al., 2014). We can expect these riddles to be solved by the experts in the future. Coming to the Internet of Things, the structural technologies involved are embedded systems, networking protocols and infrastructure, cloud computing and big data analytics. However, the research issues involved in these technologies are effectiveness in security, load balancing, scalableand interoperable environment development.

\section{Conclusion:}

The cloud and the integrated technologies are facing a lot of issues in spite of its effective application. The security, integrity and conf identiality triangle is considered as the basic quality for any medium that involves data communication (Bacon et al., 2014). Hence, a secured cloud principle should be designed with a ability to check any present issue. We insist on the development of cloud security, because all the future world technologies have adopted cloud as their medium of storage and processing, due to its provisions like scalability, fault tolerance, flexibility and utility model. We predict the cloud computing will dominate the future world of computing with increased ability, powered by the upcoming high speed internet technologies. We predict there would be maximum migration of technologies to cloud Environment. Hence, the cloud will bloom and partner with all the technologies, because the Internet of Things has itself adapted to cloud environment. As long as these integrated technologies are widespread, the cloud remains stable and established.

\begin{tabular}{|c|c|c|c|c|c|c|}
\hline & $\begin{array}{l}\text { o Technology/ } \\
\text { Sector }\end{array}$ & Proposed by & $\begin{array}{l}\text { Major area(s) of } \\
\text { Influence }\end{array}$ & $\begin{array}{l}\text { ApplicationKey } \\
\text { Methos }\end{array}$ & Features & $\begin{array}{l}\text { Integrated } \\
\text { Technology }\end{array}$ \\
\hline 1 & $\begin{array}{l}\text { Mobile Cloud } \\
\text { Computing }\end{array}$ & $\begin{array}{l}\text { Ahmed et al., } 2015 \\
\text { Khan et al., } 2014 \\
\text { Abolfazli et al., } 2014 \\
\text { Yegui et al., } 2014 \\
\text { Chen et al., 2015 } \\
\text { Aminzadeh et al., } \\
2015\end{array}$ & $\begin{array}{l}\text { Data and Network } \\
\text { Centric Performance } \\
\text { analysis, Decision } \\
\text { making in migration } \\
\text { of Application, } \\
\text { heterogeneity }\end{array}$ & $\begin{array}{l}\text { OMNet++, } 5 \mathrm{G} \text {, } \\
\text { emotion aware } \\
\text { computing }\end{array}$ & $\begin{array}{l}\text { Application Migration in runtime, } \\
\text { offloading, considers various network } \\
\text { parameters for Analysis. Overcomes } \\
\text { the issues faced by Application } \\
\text { Execution Frameworks }\end{array}$ & $\begin{array}{l}\text { Mobile } \\
\text { Computing } \\
\text { and Cloud } \\
\text { computing }\end{array}$ \\
\hline 2 & $\begin{array}{l}\text { Manufacturing \& } \\
\text { Service Sectors }\end{array}$ & $\begin{array}{l}\text { Buyya et al., } 2019 \\
\text { Marston et al., } 2011\end{array}$ & $\begin{array}{l}\text { Diffusion of Innovation } \\
\text { (DOI) Technology } \\
\text { Organization Environ- } \\
\text { ment (TOI) }\end{array}$ & $\begin{array}{l}\text { Factor Analysis, } \\
\text { conceptual Analysis, } \\
\text { Multiple Regression } \\
\text { analysis }\end{array}$ & SME (small and Medium Enterprises) & $\begin{array}{l}\text { SME and } \\
\text { Cloud } \\
\text { Computing }\end{array}$ \\
\hline 3 & Bigdata Analytics & $\begin{array}{l}\text { Marston et al., } 2011 \\
\text { Branch et al., } 2014 \\
\text { Sookhaka et al., } 2015 \\
\text { Mehdi \& Singhal, } \\
2015\end{array}$ & $\begin{array}{l}\text { Ubiquitous Computing, } \\
\text { Virtualization of Storage \& } \\
5 \text { Processing the data }\end{array}$ & $\begin{array}{l}\text { No Sql based Data } \\
\text { Access }\end{array}$ & Exascale analytics, Audit & $\begin{array}{l}\text { Scientific } \\
\text { and business } \\
\text { data analysis, } \\
\text { with cloud } \\
\text { computing }\end{array}$ \\
\hline 5 & $\begin{array}{l}\text { Healthcare } \\
\text { Technologies }\end{array}$ & $\begin{array}{l}\text { Jula et al., } 2014 \\
\text { Chen et al., } 2015 \\
\text { Sultan, } 2014\end{array}$ & Healthcare & $\begin{array}{l}\text { Wearable and } \\
\text { implanted sensors }\end{array}$ & $\begin{array}{l}\text { Ability to monitor health time to time. } \\
\text { Alert mechanism. Reliable healthcare }\end{array}$ & $\begin{array}{l}\text { Wireless } \\
\text { Sensors, Cloud } \\
\text { Computing, IoT }\end{array}$ \\
\hline 6 & File Management & Gupta, 2015 & $\begin{array}{l}\text { Private, Public and } \\
\text { community basis }\end{array}$ & Amazon EC2 cloud & $\begin{array}{l}\text { Provision to store data, Cloud based } \\
\text { VPS, FTP }\end{array}$ & Cloud Storage \\
\hline
\end{tabular}


7 Internet of Things Wang et al., 2014 Automation, Monitoring \& Sensors, actuators Aazam et al., 2014 controlling from remote end and embedded
Establish inter-operability, remote monitoring, communication and aut omation of devices with internet
Wireless sensors,

Knowledge base,

Protocols, actuators, Cloud computing, big data analytics, etc.,

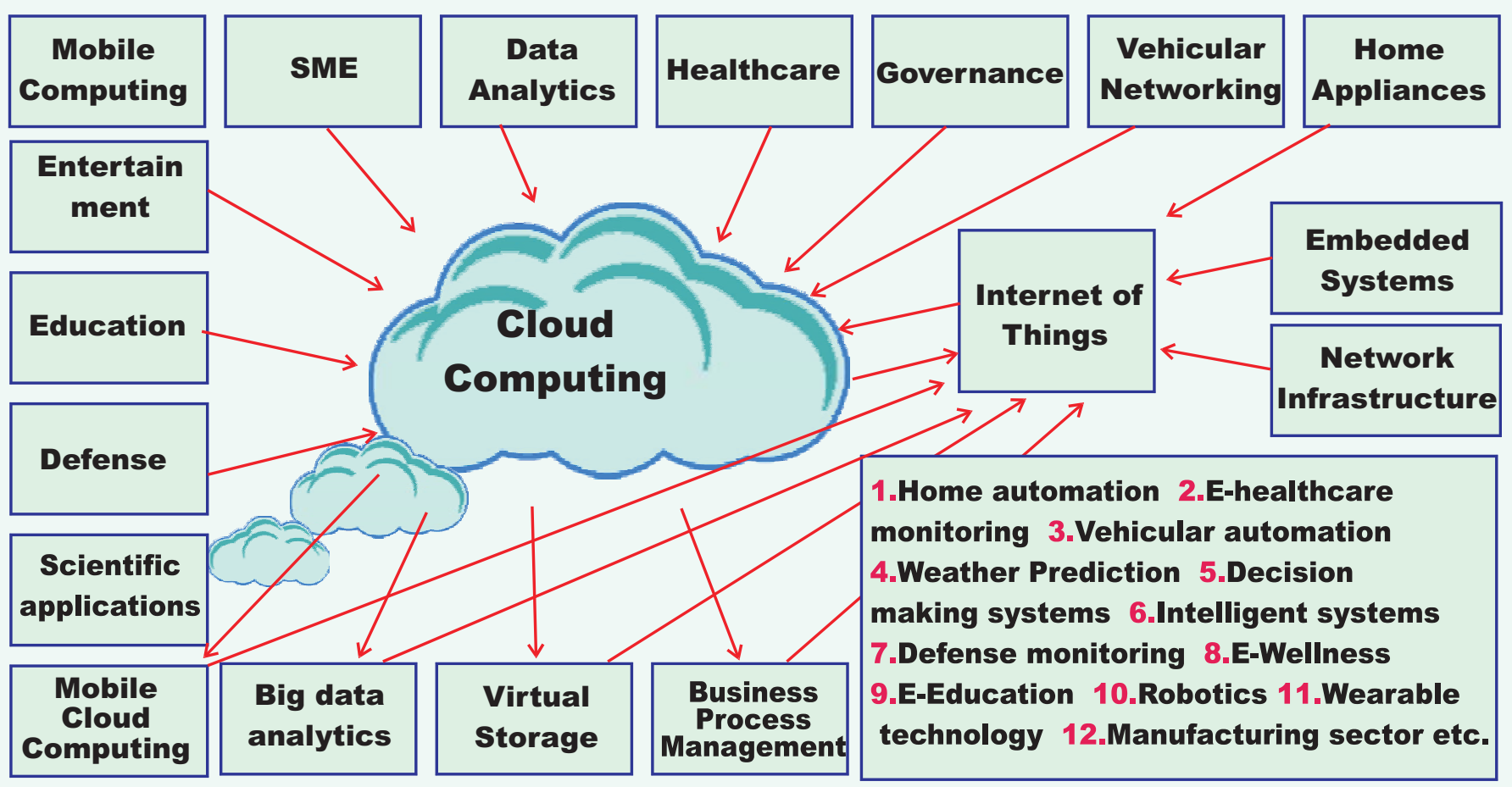

Figure- 5: cloud computing and Integrated technologies

Acknowledgements:

I recognize with thanks the sort of support; cherishing motivation and opportune direction from Dr. G .Ramakrishna Reddy .I likewise recognize the Google and the composing staff of ambient science."

\section{References:}

Aazam, M., Khan, I., Alsaffar, A.A. \& Huh, E. (2014): Cloud of Things: Integrating Internet of Things and cloud computing and the issues involved." Proceedings of Applied Sciences and Technology (IBCAST), 2014 11th International Bhurban Conference on. IEEE, p. 414-419.

Abrishami, S., Naghibzadeh, M. \& Epema, D.H.J. (2013): Deadlineconstrained workflow scheduling algorithms for Infrastructure as a Service Clouds. Fut. Gener. Comp. Sy. 29(1):158-169.

Abolfazli, S., Sanaei, Z., Ahmed, E., Gani, A. \& Buyya, R. (2014):. Cloud-based Augmentation for Mobile devices: Motivation, Taxonomies, and Open Challenges. IEEE Commun.Surv. Tut., 16(1):337-368

Abolfazli, S., Sanaei, Z., Tabassi, A., Rosen, S., Xchanging, Gani, A. \& Khan, S.U. (2015): Cloud Adoption in Malaysia: Trends, Opportunities, and Challenges. Cloud Comp. IEEE., 2.1: 6o-68

Ahmed, E., Akhunzada, A., Whaiduzzaman, Md., Gani, A., Hamid, S.H.A. \& Buyya, R. (2015): Network-centric performance analysis of runtime application migration in mobile cloud computing. Simul. Model. Prac.Th., 50:42-56.

Aminzadeh, N., Sanaei, Z. \& Hamid, S.H.A. (2015): Mobile storage augmentation in mobile cloud computing: Taxonomy, approaches, and open issues. Simul.Model.Prac.Th., 50: 96108.

Aminzadeh, N., Sanaei, Z. \& Hamid, S.H.A. (2015): Mobile storage augmentation in mobile cloud computing: Taxonomy, approaches, and open issues. Simul. Model. Prac.Th., 50: 96108.

Bacon, J., Eyers, D. Pasquier, T., Singh, J., Papagiannis, I. \& Pietzuch, P. (2014): Information flow control for secure cloud computing. Net. Ser. Manag., IEEE Transac., 11(1): 76-89.

Bitam, S., Mellouk, A. \& Zeadally, S. "VANET-cloud:a generic cloud computing model for vehicular Ad Hoc networks." Wirel. Comm. IEEE, 22(1): 96-102.

Branch, R. , Tjeerdsma, H. , Wilson, C. , Hurley, R. \& McConnell, S. (2014): Cloud Computing and Big Data: A Review of Current Service Models and Hardware Perspectives. J. Softw. Eng. Applic., 7: 686-693

Bruneo, D. (2014): A stochastic model to investigate data center performance and qos in iaas cloud computing systems. $\underline{\text { Parall. }}$ Distr. Sy. IEEE Transac., 25(3):560-569.

Buyya, R., Yeo, C.S. \& Venugopal, S. (20o8): Market-oriented cloud computing: Vision, hype, and reality for delivering it services as computing utilities." High Performance Computing and Communications, 2008. HPCC'o8. 10th IEEE International Conference on. Ieee, 2008.

Buyya, R., Yeo, C.S., Venugopal, S., Broberg, J. \& Brandic, I. (2009): "Cloud computing and emerging IT platforms: Vision, hype, and reality for delivering computing as the 5 th utility. Fut. 
Gener. Com. Sy., 25(6): 599-616.

Chen, M., Y. Zhang, Y. Li, M. \& Alamri, H.A. (2015): AIWAC: affective interaction through wearable computing and cloud technology. Wirel. Commun. IEEE, 22(1):20-27.

Chen, .M, Zhang, Y., Li, Y., Mao, S. \& Leung, V.C.M. (2015): Emc: Emotion-aware mobile cloud computing in 5g. Netw. IEEE, 29(2):32-38.

Dong, X., Yu, J., Luo, Y. Chen, U., Xue, G. \& Li, M. (2014): Achieving an effective, scalable and privacy-preserving data sharing service in cloud computing. Comp. Secur., 42:151-164.

Gupta, U. (2015): Survey on security issues in file management in cloud computing environment. arXiv preprint, arXiv:1505.00729.

Jain, N., Menache, I., Naor, J. \& Yaniv, J. (2014): A truthful mechanism for value-based scheduling in cloud computing. Th. Compu. Sy., 54(3):388-406.

Jula, A., Sundararajan, E. \& Othman, Z. (2014): Cloud computing service composition: A systematic literature review. Expert Sy.Appl., 41(8):3809-3824.

Khan, A.R., Othman, M., Madani, S.A., \& Khan, S.U. (2014): A survey of mobile cloud computing application models. Commun. Surv. Tut. IEEE, 16(1):393-413.

Marston, S., Li, Z., Bandyopadhyay, S., Zhang, J. \& Ghalsasi, A. (2011): Cloud computingThe business perspective. Dec. Supp. Sy., 51(1): 176-189.

Mehdi, B. \& Singhal, M. (2015): The Role of Cloud Computing Architecture in Big Data; in Pedrycz, W \& Chen, S.M ed.Information Granularity, Big Data, and Computational Intelligence. Springer International Publishing. P. 275-295.
Mell, P. \& Grance, T. (2011): The NIST definition of cloud computing. Spl. Publication. 800-145

Oliveira, T., Thomas, M. \& Espadanal, M (2014): Assessing the determinants of cloud computing adoption: An analysis of the manufacturing and services sectors. Inform. Manag., 51(5):497-510.

Sookhaka, M., Gani, A., Khanb , M.K., \& Buyya, R. (2015): Dynamic remote data auditing for securing big data storage in cloud computing. Inform. Sci., ooo:116

Sultan, N (2014): Making use of cloud computing for healthcare provision: Opportunities and challenges. Int. J. Inform. Manag.,34(2): 177-184.

Wang, C., Bi, Z. \& Xu, L.D. (2014): IoT and cloud computing in automation of assembly modeling systems. Indus. Inform., IEEETransac., 10(2):1426-1434.

Wei, L., Zhu, H., Cao, Z., Dong, X., Jia, W., Chen, Y. \& Vasilakos, A.V. (2014): Security and privacy for storage and computation in cloud computing. Inform. Sci., 258:371-386.

Whaiduzzaman, Md., Sookhak, M., Gani, A. \& Buyya, R. (2014): A survey on vehicular cloud computing."J. Netw. Comp. Appl., 40:325-344.

Yegui, C., Yu, F.R. \& Bu, S. (2014): Cloud computing meets mobile wireless communications in next generation cellular networks. Netw. IEEE, 28(6): 54-59.

Zhan, Z., Lui, X., Gong, Y. \& Zhang, J. (2015): Cloud computing resource scheduling and a survey of its evolutionary approaches. ACM Comp. Sur., (CSUR), 47(4): 63. 